\section{PSICOLOGIA IBEROAMERICANA}

\section{Psicología lberoamericana}

ISSN: 1405-0943

psicología.iberoamericana@uia.mx

Universidad Iberoamericana, Ciudad de

México

México

Limón González, José Briam; Rocha Sánchez, Tania Esmeralda

Creencias y Actitudes Sexistas de Trabajadores en Empresas Mexicanas: Un Estudio Exploratorio

Psicología Iberoamericana, vol. 19, núm. 2, julio-diciembre, 2011, pp. 55-66

Universidad Iberoamericana, Ciudad de México

Distrito Federal, México

Disponible en: http://www.redalyc.org/articulo.oa?id=133921440007

Cómo citar el artículo

- Número completo

- Más información del artículo

- Página de la revista en redalyc.org

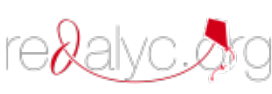

Sistema de Información Científica

Red de Revistas Científicas de América Latina, el Caribe, España y Portugal

Proyecto académico sin fines de lucro, desarrollado bajo la iniciativa de acceso abierto 


\title{
Creencias y Actitudes Sexistas de Trabajadores en Empresas Mexicanas: Un Estudio Exploratorio
}

\author{
Sexist Beliefs and Attitudes of Workers in Mexican Companies: \\ An Exploratory Study
}

José Briam Limón González* Tania Esmeralda Rocha Sánchez FACULTAD de PSICOLOGÍa Universidad Nacional Autónoma de México, CD. De México

\section{RESUMEN}

Con el propósito de analizar las creencias y actitudes sexistas hacia líderes de empresas, se realizó un estudio de carácter exploratorio con 90 trabajadores, a quienes se preguntó las características que identificaban en líderes de ambos sexos y las actitudes que tienen hacia ellos. Se realizó un análisis de contenido de las respuestas bajo la perspectiva del sexismo ambivalente y la teoría de la congruencia con el rol de género. Se encontró que, efectivamente, el conjunto de creencias y actitudes hacia los y las líderes incorpora elementos prescriptivos sobre el rol de género, aludiendo tanto a lo que es normativo como al reconocimiento de las incongruencias con dicho rol según el contexto cultural. Dados los resultados, es indispensable innovar en formas de conceptuar y operacionalizar el sexismo, reconociendo su ambivalencia (hostil y benevolente) y las diversas formas en las que puede manifestarse en el contexto laboral.

Descriptores: trabajo, género, estereotipos, actitudes, liderazgo

\section{ABSTRACT}

In order to analyze the beliefs and attitudes toward corporate leaders, we made an exploratory study with 90 workers who were asked to identify the characteristics of leaders of both sexes and attitudes towards them. A content analysis from the perspective of ambivalent sexism and role congruity theory was conducted. It was found that indeed the set of beliefs and attitudes towards leaders includes prescriptive elements of the gender role, referring to what is normative and recognition of inconsistencies with that role according to the cultural context. Given the results, it is essential to innovate in ways to conceptualize and operationalize the ambivalent sexism (hostile and benevolent) and the various ways in which this can be appear in organizational context.

Key words: work, gender, stereotypes, attitudes, leadership 


\section{INTRODUCCIÓN}

De acuerdo a los datos mostrados por el Instituto Nacional de Estadística Geografía e Informática, mediante la Encuesta Nacional de Ocupación y Empleo (INEGI, 2007), en México, $84.2 \%$ de empleadores y patrones a nivel nacional fueron hombres; la tasa de participación económica de los hombres en general fue de $75.5 \%$ e, independientemente del sector laboral analizado, las mujeres percibieron un ingreso menor que el de los hombres por las mismas actividades. Además, en concordancia con lo señalado, la revista $C N N$ Expansión (2010) indica que las mujeres sólo dirigen $3 \%$ de las 500 empresas más importantes de México (15 empresas). Esto también se hace evidente en las presidencias de organismos empresariales, ya que, de acuerdo a Zabludovsky (2007), 95\% de los presidentes de las cámaras que constituían la Confederación Patronal de la República Mexicana (COPARMEX) y 94\% de los que constituían la Confederación de Cámaras Industriales (CONCAMIN) en ese mismo año, fueron hombres. Inclusive, datos del Índice de Disparidad entre Géneros del Foro Económico Mundial (FEM, 2009) muestran que México descendió dos posiciones en dicho listado con respecto al 2008, lo cual lo ubica en el lugar 99 de 134 países. En este índice se evalúa el buen uso y reparto de los recursos y oportunidades entre la población femenina y masculina de cada país, independientemente de los niveles generales de dichos recursos y oportunidades.

Datos como los anteriores muestran de forma contundente que hombres y mujeres no tienen la misma participación en puestos de liderazgo, tanto en el sector público como en el privado, lo cual tiene serias implicaciones para el desarrollo profesional de las mujeres.

Esto toma particular relevancia en el contexto de una cultura como la mexicana, en la que, a decir de Díaz-Guerrero (1990), las bases para la lógica específica de los grupos se estructura a partir de las premisas socioculturales que indican a hombres y mujeres lo que deben "pensar, sentir y hacer" en función de la "superioridad del hombre" y la "abnegación de la mujer". Dichas afirmaciones se convierten en el eje de las relaciones interpersonales que marcan los lineamientos y roles específicos para un sexo u otro. De este modo, hay una serie de creencias sobre el papel que deben cumplir ambos sexos en cada una de las esferas sociales, tales como la familia, los amigos o el trabajo. En este último, existe una división sexual sustentada en las creencias sobre los roles de género, que asigna a las mujeres y a los hombres puestos de menor y mayor remuneración económica, respectivamente.

La discriminación contra las mujeres en el trabajo no es algo nuevo y mucho menos algo resuelto. Sin embargo, la sociedad actual no reporta abiertamente ejercer esta discriminación, entre otras cosas debido a que los planteamientos y esfuerzos por implementar una equidad de género han llevado a las personas a responder de una forma "socialmente deseable" respecto a esta temática, por lo que resulta más complicado evaluar dicho fenómeno. Inclusive, de acuerdo a Eagly y Mladinic (1994), las mujeres ahora son estereotipadas de una forma más positiva que los hombres, lo cual representaría una paradoja en términos de un modelo de prejuicio que señala la subordinación de la mujer como elemento constitutivo de toda sociedad. A decir de Biglia (2003b), el dominio sobre las mujeres ha evolucionado en el ámbito social, asumiendo formas más sutiles de control generalizado, en ocasiones más efectivas y más difíciles de desenmascarar. Esto ha sido denominado como sexismo moderno e implica la coexistencia tanto de los sentimientos negativos tradicionales hacia las mujeres, como la aceptación de elementos igualitarios entre un sexo y otro, en tanto guarden correspondencia con los aspectos socialmente deseables para hombres y mujeres (Swim, Aikin, Hall \& Hunter, 1995). Este proceso, como sugiere Fernández (2000), conduce a la imposibilidad de reconocer e incluso ignorar la discriminación por sexo que se presenta en distintos ámbitos de convivencia social, ya que, al estar sustentada en percepciones positivas sobre los sexos, no parece denotar el componente evaluativo negativo tradicional del sexismo.

Al respecto, Swim, Aikin, Hall y Hunter (1995) citan un sondeo de opinión Gallup realizado en los Estados Unidos de América en 1990, según el cual 43\% de los hombres y $54 \%$ de las mujeres preferían tener en su trabajo un supervisor que una supervisora. En cambio, apenas $15 \%$ de ellos y $12 \%$ de ellas dijo preferir una supervisora.

En otro estudio, Ryan, Haslam, Hersby y Bongiorno (2007) cuestionaron a 1602 trabajadores sobre 
las características de un líder que hace las cosas bien, un líder que hace las cosas mal, un hombre y una mujer. El resultado más sobresaliente fue una fuerte asociación ( $r=0.82, p<.01)$ entre el estereotipo femenino y un líder que hace las cosas mal.

Otra manifestación sutil de sexismo en el trabajo implica asociar determinadas conductas como específicas de hombres o mujeres líderes. En una revisión documental, Eagly y Johnson (1990) concluyeron que las mujeres líderes tienden más a adoptar un estilo democrático y optan menos por uno autocrático que los hombres. El sexismo radica en que asociar exclusivamente un determinado estilo con cada sexo implicaría una evaluación diferencial de los líderes cuando manifiesten un estilo contrario al esperado (por ejemplo, hombre democrático o mujer autocrática).

Los hallazgos de las investigaciones mencionadas enfatizan la influencia que tienen los estereotipos y las actitudes hacia los roles de género sobre una serie de aspectos que involucran, tanto evaluaciones negativas de la participación de mujeres en puestos de liderazgo, como toma de decisiones que pueden afectar su ascenso a dichos puestos (discriminación).

Una alternativa para explicar lo señalado reside en la propuesta del sexismo ambivalente, de Glick y Fiske (1996). Dichos autores indican que las actitudes positivas hacia las mujeres se dan sólo en algunas áreas que se vinculan a su rol de género tradicional (cuidado, comprensión, cariño), las cuales en sí mismas son una forma de prejuicio en la medida que sirven para justificar y mantener la subordinación de las mujeres. A estas actitudes positivas hacia las mujeres por desempeñar un rol de género tradicional les denominaron sexismo benevolente. Por otra parte, se consideró que aunado al componente benevolente se presentaba una actitud negativa o de antipatía hacia las mujeres por demandar, como lo menciona Izquierdo (2003), el carácter de ciudadanas-individuo en la participación social, es decir, por externalizar las peticiones del "ideario feminista". A dicha dimensión Glick y Fiske la nombraron sexismo hostil. Lo que se puede apreciar en estas escalas es que el sexismo se presenta cuando las mujeres son delimitadas a la función de cuidadoras, o cuando son prejuzgadas por pretender asumir el rol de protectoras o proveedoras, ya que dentro de un esquema de poder estructural masculino esto im- plica el rompimiento con las relaciones de complementariedad entre los géneros, que como mencionan estos autores, se asienta sobre la base del control en los hombres y de las relaciones en las mujeres. Años más tarde, estos autores (1999) consideraron que tales dimensiones también podían hacerse presentes en la dirección inversa, esto es, como sexismo hostil y benevolente hacia los hombres. En este caso, el sexismo hostil se haría evidente en la medida que a los hombres se les asociara con el rol de género masculino negativo (abuso del poder), mientras que el sexismo benevolente correspondería al cumplimiento con el rol de género masculino positivo (protección). En el caso de la escala de sexismo hostil hacia los hombres, resulta importarte identificar que el sexismo no se origina en la transgresión de roles de género, es decir, los hombres no son juzgados por pretender realizar "actividades femeninas", sino por considerar que quieren mantener el control y poder sobre las mujeres.

En el mismo sentido, Eagly y Karau (2002) plantean la teoría de la congruencia con el rol de género, en la que señalan que el prejuicio contra un individuo estará en función del grado de incongruencia percibida entre el rol que esté desempeñando y el rol de género prescrito para su sexo. Así, cuando un hombre desempeñe una actividad tradicionalmente catalogada como femenina, tenderá a ser más prejuiciado tanto positiva como negativamente que una mujer realizando la misma actividad. En el caso de las mujeres, GarcíaRetamero y López-Zafra (2006) encontraron que son evaluadas desfavorablemente cuando realizan una actividad considerada tradicionalmente masculina, como lo es el liderazgo empresarial. Concretamente, sus resultados mostraron que los éxitos de las líderes se atribuyeron a elementos externos tales como la suerte, mientras que sus fracasos se asociaron a aspectos internos como la falta de capacidad.

De tal suerte que conviene analizar la discriminación sexista en el trabajo a partir de una serie de creencias prescriptivas tanto positivas como negativas sobre el desempeño de hombres y mujeres en dicho ámbito; es decir, no sólo retomar los aspectos hostiles y evidentes de la división sexual del trabajo, sino también recurrir a los aspectos benevolentes o encubiertos que se pueden desarrollar en el mismo. 
Por lo tanto, entender cómo es que hombres y mujeres son segregados y tratados de formas diferenciales en el contexto laboral, salarial, etcétera, exige no sólo ver la manifestación externa de este proceso discriminatorio, sino entender su origen desde las creencias y actitudes que lo sostienen. Aunado a ello, el estudio de esta problemática, bajo una mirada crítica, hace evidente que el asunto del sexismo no se limita en el conjunto de creencias y prejuicios contra las mujeres, sino que, precisamente porque el género es un asunto estructural y relacional, afecta e incide en la vida tanto de hombres como de mujeres y en diferentes esferas. Esto es, los estereotipos, los prejuicios y la discriminación sexista se pueden presentar para ambos géneros. En consecuencia, es desde esta misma perspectiva que se evidencia cómo pese a que impacta a ambos, las formas, las razones y circunstancias, e incluso las consecuencias del sexismo, llegan a afectar de forma diferente tanto a hombres como a mujeres, en la medida en la que las premisas culturales que delimitan las identidades de ambos pueden ser más severas o limitadoras hacia un sexo u otro, en función del escenario social.

En México se carece de investigaciones derivadas de los planteamientos del sexismo ambivalente y de la teoría de la congruencia con el rol de género que aborden la escasa participación de las mujeres en puestos ejecutivos, lo cual puede resultar en una aproximación más precisa al fenómeno, al integrar tanto los aspectos evidentes como los encubiertos de la problemática. En el mismo sentido, los inventarios de sexismo ambivalente desarrollados por Glick y Fiske (1996, 1999), si bien están orientados a la evaluación del sexismo en términos de interacciones sociales, que reflejan el componente estructural de las creencias estereotípicas sobre los sexos, no permiten identificar los aspectos específicos de dicho sexismo en el escenario laboral. De acuerdo a Glick, Diebold, Bailey-Werner y Zhu (1997), para los hombres sexistas existen grupos de mujeres que merecen un tratamiento hostil, mientras que otros grupos de mujeres merecen ser tratadas con benevolencia. Sobre lo anterior cabría preguntar ¿cuál es la percepción que se tiene de las líderes? ¿Se contempla a las líderes en el grupo de mujeres que merecen un trato hostil o uno benevolente? ¿Los líderes son evaluados bajo la misma premisa en las empresas?
En cuanto a la aplicación práctica de la identificación del sexismo en escenarios organizacionales, algunos autores como Grosser y Moon (2005) señalan que al incorporar la evaluación del sexismo moderno en las políticas, planes, programas y actividades en las empresas, redunda en mejoras en la gestión del capital humano, así como consecuencias positivas en el plano económico y de imagen corporativa a largo plazo.

Por ello, el propósito de esta investigación fue identificar las creencias y predisposiciones actitudinales que se tienen hacia hombres y mujeres líderes en empresas de México, partiendo del supuesto de que las creencias y las actitudes son la base de un proceso sexista tanto hostil como benevolente, y que éstas están altamente vinculadas al contexto cultural. Conviene resaltar que el presente estudio, al constituir un abordaje exploratorio de la problemática, pretende incorporar una nueva mirada al análisis del sexismo en las empresas, y por ende sus resultados no buscan, en primera instancia, la generalización que la integración y replica de futuras investigaciones ofrecerán, con escalas estandarizadas y evaluaciones controladas emanadas de este planteamiento.

\section{MÉTODO}

\section{Participantes}

Se asistió a organizaciones laborales del sector productivo asociado a la manufactura de la zona centro de México. Dentro de las cuales se realizó un muestreo no probabilístico de tipo intencional y se trabajó con 48 hombres y 42 mujeres de distintos niveles jerárquicos que aceptaron participar voluntariamente. El promedio de edad fue de 36 años (D.E. $=4.6$ años). La moda de escolaridad fue de preparatoria y los participantes se desempeñaban fundamentalmente como supervisores o mandos medios (72\%).

\section{Técnica}

Para identificar las creencias y actitudes sexistas hacia líderes de empresas se elaboró un cuestionario de siete preguntas abiertas ex profeso para indagar en torno a características y prejuicios generales hacía líderes de empresas. Del total, tres fueron distractoras (¿qué líderes de empresas conoce?). En la parte final venían preguntas generales sobre su sexo, el puesto que desempeñaban al momento de la aplicación, la edad y la escolaridad. 
Para abordar el aspecto de creencias sobre líderes de ambos sexos, se aplicaron las siguientes preguntas:

a) ¿Cuáles son las características positivas y negativas de hombres líderes de empresas?

b) ¿Cuáles son las características positivas y negativas de mujeres líderes de empresas?

En el caso de las predisposiciones actitudinales, se plantearon las citadas a continuación:

a) ¿Qué le gusta o disgusta de una mujer líder de empresa?

b) ¿Qué le gusta o disgusta de un hombre líder de empresa?

\section{Procedimiento}

Se entregó el cuestionario de preguntas abiertas respecto a las creencias y actitudes a cada trabajador y se les indicó que respondieran en función de su propia experiencia y con la mayor sinceridad posible. El tiempo que llevó a los trabajadores contestar dicho cuestionario fue de 15 minutos, aproximadamente, y una vez que finalizaron se les garantizó la confidencialidad de sus respuestas. Se recolectaron el total de aplicaciones y se procedió a la transcripción de las mismas al programa ATLAS-ti (v.5.0), en el que se obtuvieron diagramas de las características y actitudes hacia líderes de distinto sexo con el fin de realizar un análisis de contenido de los datos bajo la perspectiva del sexismo hostil y benevolente en el trabajo.

En ese sentido, se formaron categorías en la que se identificaron los aspectos negativos caracterizados por una actitud dominante y desafiante frente a hombres y mujeres que implica una percepción negativa del cumplimiento o no de los roles de género prescritos (sexismo hostil). Un ejemplo de esta categoría se identifica en la siguiente afirmación: "En el fondo, las mujeres feministas pretenden que la mujer tenga más poder que el hombre".

Por otro lado, se formaron categorías caracterizadas por el conjunto de creencias y actitudes positivas que estereotipan y limitan los roles de género de hombres y mujeres en tanto consideran una visión complementaria de los sexos (sexismo benevolente). El ejemplo correspondiente a esta categoría es el siguiente:
"Las mujeres deben ser queridas y protegidas por los hombres". Además, con base en las categorías anteriores, se identificaron aspectos de congruencia e incongruencia con el rol de género tradicional en función de lo esperado culturalmente para hombres y mujeres.

\section{RESULTADOS}

A continuación se muestran los resultados cuyo fin fue explorar las características y manifestaciones de creencias y actitudes sexistas que tienen los trabajadores mexicanos hacia líderes de empresas, en términos de lo que consideran deben ser y hacer en función de su rol de género.

Respecto a las características de los líderes, las respuestas de los participantes se clasificaron a partir de las características positivas o negativas asociadas a los hombres y a las mujeres en el contexto del liderazgo. Se retomaron como criterios de categorización tanto el sexismo hostil como el benevolente, que parten de la perspectiva de sexismo ambivalente y la percepción de congruencia con el rol de género tradicional.

En el caso de los hombres líderes, se manifestaron dos tipos de creencias negativas: $a$ ) vinculadas al rol de género masculino tales como autoritarismo (son prepotentes), la corrupción (realiza estrategias poco éticas para alcanzar sus objetivos), el machismo (discriminan a las mujeres), el abuso (abusan de su poder para sacar provecho) y el acoso (por tener el control quieren obtener otras cosas de las mujeres), las cuales se vinculan al sexismo hostil, y $b$ ) las femeninas, dentro de las cuales sólo figuró la metrosexualidad (son muy vanidosos). En cuanto a las creencias positivas, sólo se identificaron aquellas asociadas al rol de género masculino tales como carisma (son carismáticos y cautivadores), dinamismo (son muy ágiles y siempre están buscando que hacer), responsabilidad (son responsables con sus tareas) e inteligencia (son inteligentes en general), pertenecientes a la configuración del sexismo benevolente. En la figura 1 se puede apreciar que se presentaron más citas para las creencias negativas (61 menciones) que para las positivas (46 menciones) sobre los hombres líderes.

En cuanto a las creencias sobre mujeres líderes, se encontró una mayor variabilidad en las respuestas de los participantes, ya que se identificaron un mayor número de categorías (32), siendo 22 para las creencias 
Figura 1. Diagrama de las creencias positivas y negativas sobre los líderes reportadas por los trabajadores

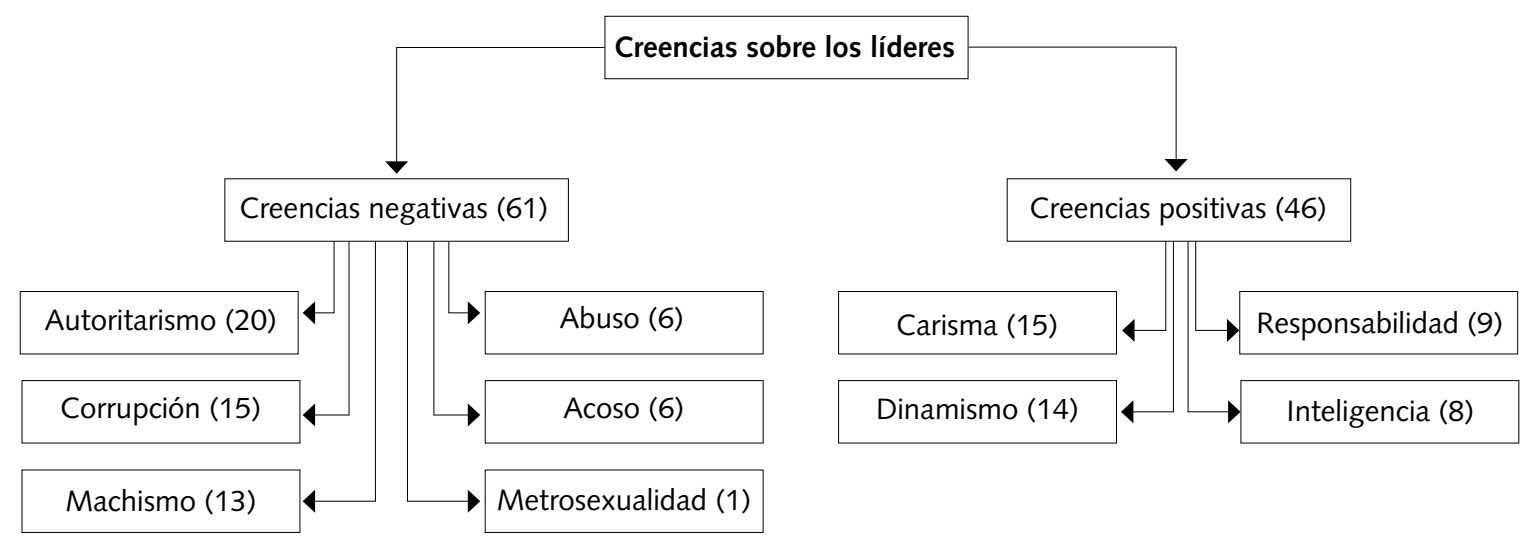

positivas y 10 para las negativas. Además, las citas asociadas a las creencias positivas tuvieron una frecuencia de 138, y para las creencias negativas la frecuencia fue de 69 (véase figura 2).

Dentro de las creencias negativas, se encontraron aquellas asociadas al rol de género femenino en su manifestación de sexismo hostil, como por ejemplo ser emocionales negativas (histéricas y desesperadas), autoritarias (son mandonas, prepotentes y abusan del poder) e incongruentes con el rol tradicional (prefieren el trabajo que a su familia); también se identificaron aquellas características vinculadas al sexismo benevolente, tales como ser débiles de carácter (son confiadas, vulnerables y les falta ser más duras), y, finalmente, las neutras como el nepotismo (mezclan la amistad con el trabajo).

En lo que respecta a las creencias positivas de las mujeres líderes, también se presentaron las congruentes con el rol de género femenino sustentadas en un sexismo benevolente, como el tener buenas relaciones (es alguien a quien puedes contarle tus problemas), ser comprensivas (son sensibles y te comprenden) o tener habilidad verbal (tienen mucha facilidad de palabra). Además se observó una serie de creencias vinculadas al rol de género masculino, las cuales no se identifican como sexistas, tales como ser decididas (luchan por sus metas para salir adelante) o independientes. Por último, hubo una mayor identificación de características vinculadas a un plano neutral como la responsabilidad, la eficacia y la organización. El total de categorías para las creencias sobre las mujeres líderes se puede apreciar en la figura dos.

A continuación se presentan los resultados del análisis de las actitudes sexistas. En primer lugar se presentan las actitudes hacia los líderes y posteriormente hacia las líderes.

Siguiendo la lógica de los resultados para las creencias sexistas, se observaron actitudes negativas y actitudes positivas hacia los líderes en términos del cumplimiento de los estereotipos de género. En la figura 3 se puede ver que se presentaron 11 categorías para las actitudes negativas y 13 para las actitudes positivas. Las frecuencias para cada una de ellas fueron 56 menciones y 68 , respectivamente.

En cuanto a las actitudes negativas hacia líderes, todas las categorías correspondieron al sexismo hostil hacia los hombres, es decir, a los trabajadores les disgusta que un líder sea autoritario, abuse del poder (que saque provecho de los demás), discrimine a las mujeres (que crean que piensan más que las mujeres y las quieran hacer menos), sea individualista (que sólo miran por su propio beneficio), su agresividad (que sean enojones y reaccionen agresivamente), no tomen en cuenta al personal y que se preocupen exclusivamente por el trabajo.

Para las actitudes positivas predominaron las asociadas al rol de género masculino identificadas con el sexismo benevolente, por ejemplo, a los trabajadores les gusta que los líderes sean carismáticos (que tienen todas las cualidades para lograr que tanto hombres como 
Figura 2. Diagrama de las creencias positivas y negativas sobre las líderes reportadas por los trabajadores

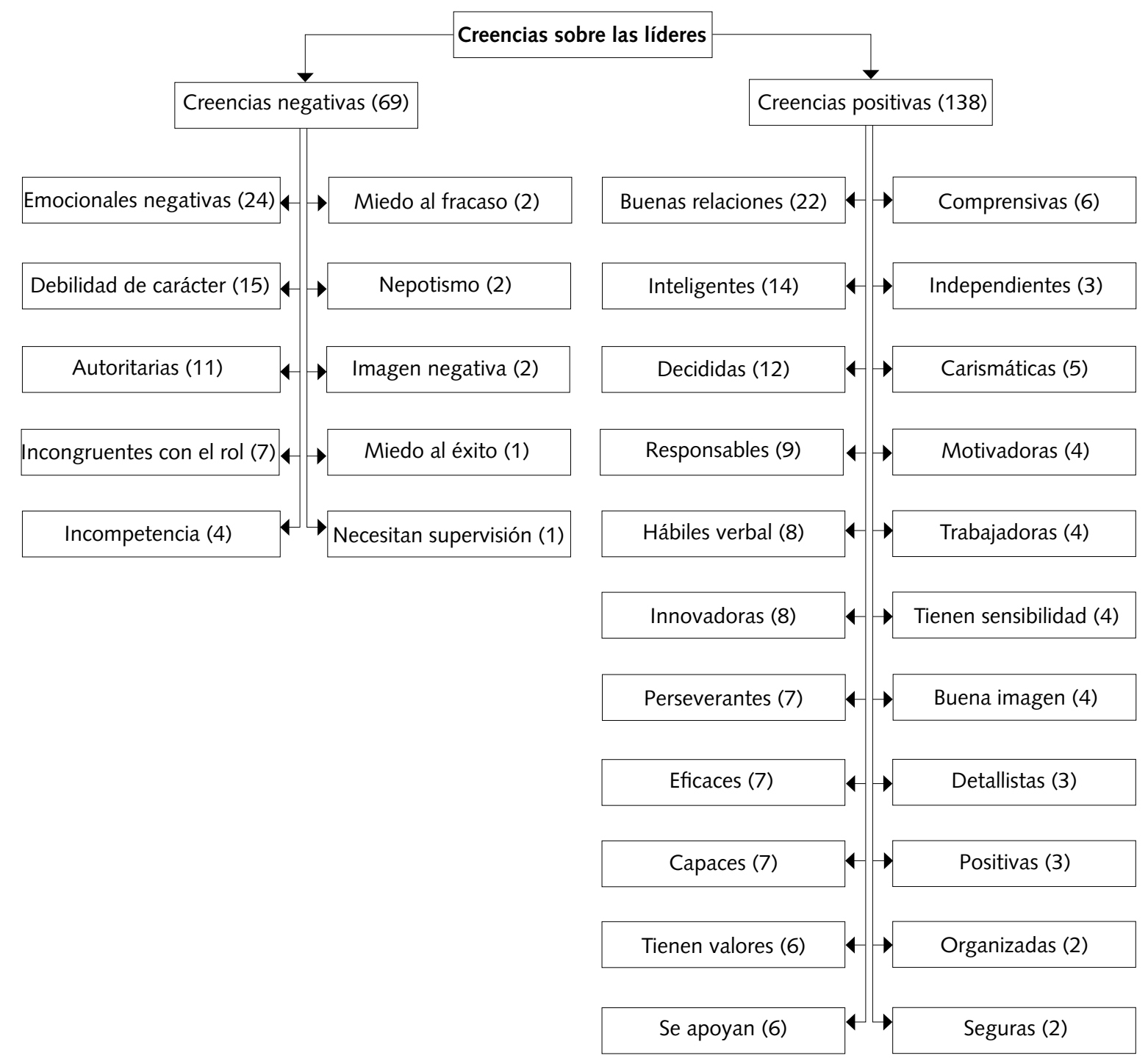

mujeres los sigan), que tengan fuerza de carácter (que sean fuertes y dominantes), que no sean sentimentales (que no se dejan llevar por sentimentalismos) ni hormonales (que no son afectados por su ciclo hormonal). algunos aspectos de los líderes que fueron asociados a categorías neutrales independientes del rol de género, como ser entregados al trabajo, proactivos (les gusta anticiparse a las cosas) o atractivos (véase figura 3). 
Figura 3. Diagrama de las actitudes positivas y negativas hacia los líderes reportadas por los trabajadores

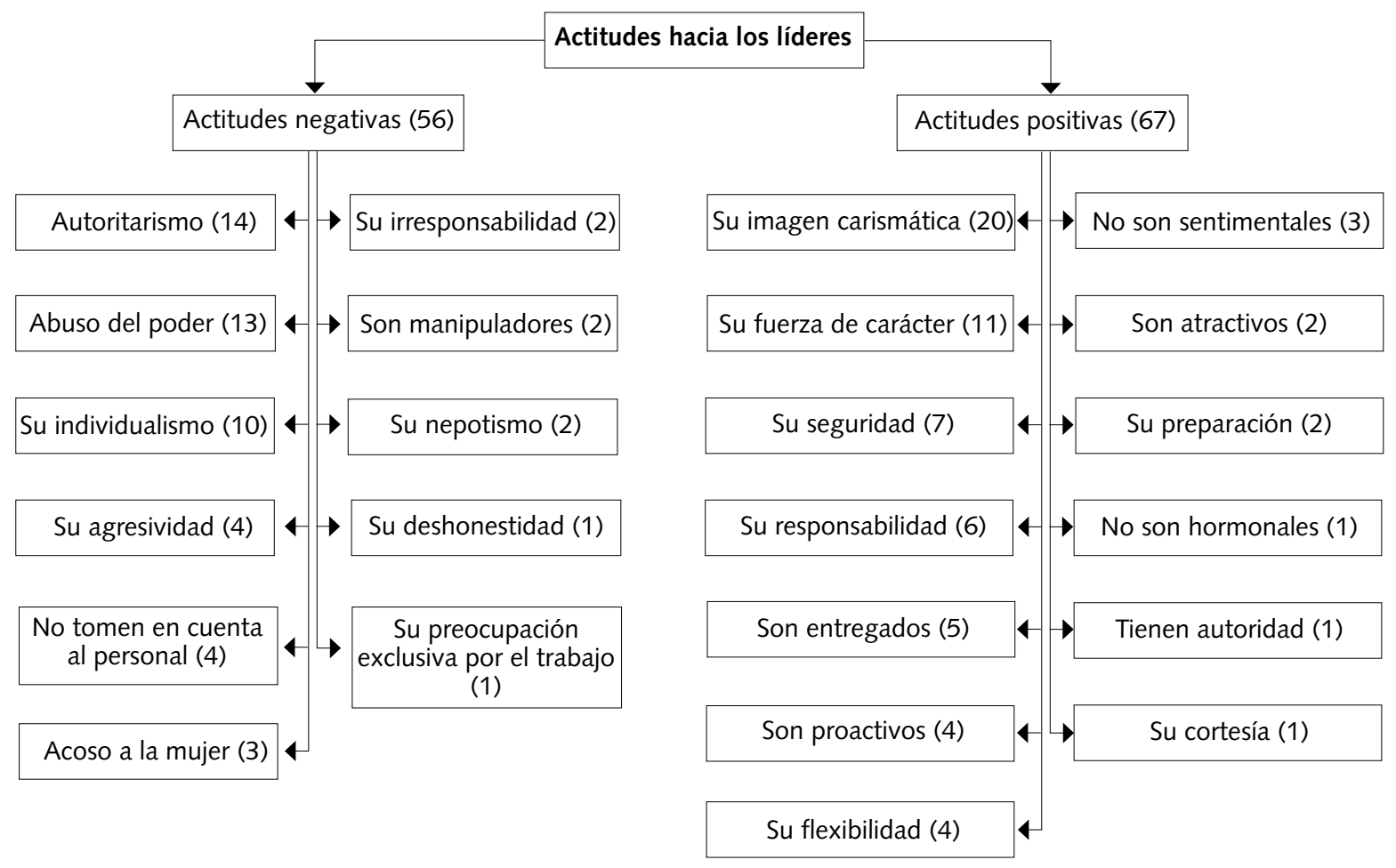

En el caso de las actitudes hacia las líderes, se presentaron más categorías positivas (17) que negativas (15). No obstante, la diferencia en las frecuencias de mención es más evidente, ya que se presentaron 96 menciones para el caso de las actitudes positivas y sólo 66 para las actitudes negativas hacia las líderes (véase figura 4). En lo que respecta a las actitudes negativas hacia las líderes, se evidencia que a los trabajadores les disgusta que las mujeres tengan características no asociadas tradicionalmente con su rol de género, tales como el autoritarismo (que sean dictadoras y no permitan otras opiniones), el egocentrismo (que se crean mucho y sólo vean por sus propios intereses) o competir con los hombres (que traten de ganarle a los hombres) aspectos manifiestos del sexismo hostil. También les disgusta que tengan características asociadas tradicionalmente a su rol de género, como por ejemplo ser manipulables (que se dejan manipular inclusive por otros hombres), relaciones laborales negativas (que se meta de más en la vida de sus trabajadores) o su sensibilidad (que por su sensibilidad a veces no sea escuchada en serio).

Por último, las actitudes positivas hacia las líderes denotaron, en primer lugar, el gusto por el buen trato que tienen las mujeres (que sean cálidas y traten bien a la gente), o su compromiso con el trabajo (que sean muy dedicadas y comprometidas con lo que hacen), aspectos vinculados a un rol femenino tradicional que forma parte de un sexismo benevolente. Posteriormente se presentan una serie de categorías vinculadas al rol de género masculino positivo tales como la capacidad (que son muy capaces al hacer las cosas), el carácter firme (son decididas y tienen un carácter fuerte) y su seguridad (que son muy seguras de sí mismas). Además, se encontró que algunos aspectos que generaron actitudes negativas también generan actitudes positivas hacia las líderes, dentro de éstos se ubican el uso del físico (que con su belleza puede abrir caminos o cerrar pactos que un hombre no podría) y la sensibilidad (que al ser más sensibles dan un trato más humano a los otros). 
Figura 4. Diagrama de las actitudes positivas y negativas hacia las líderes reportadas por los trabajadores

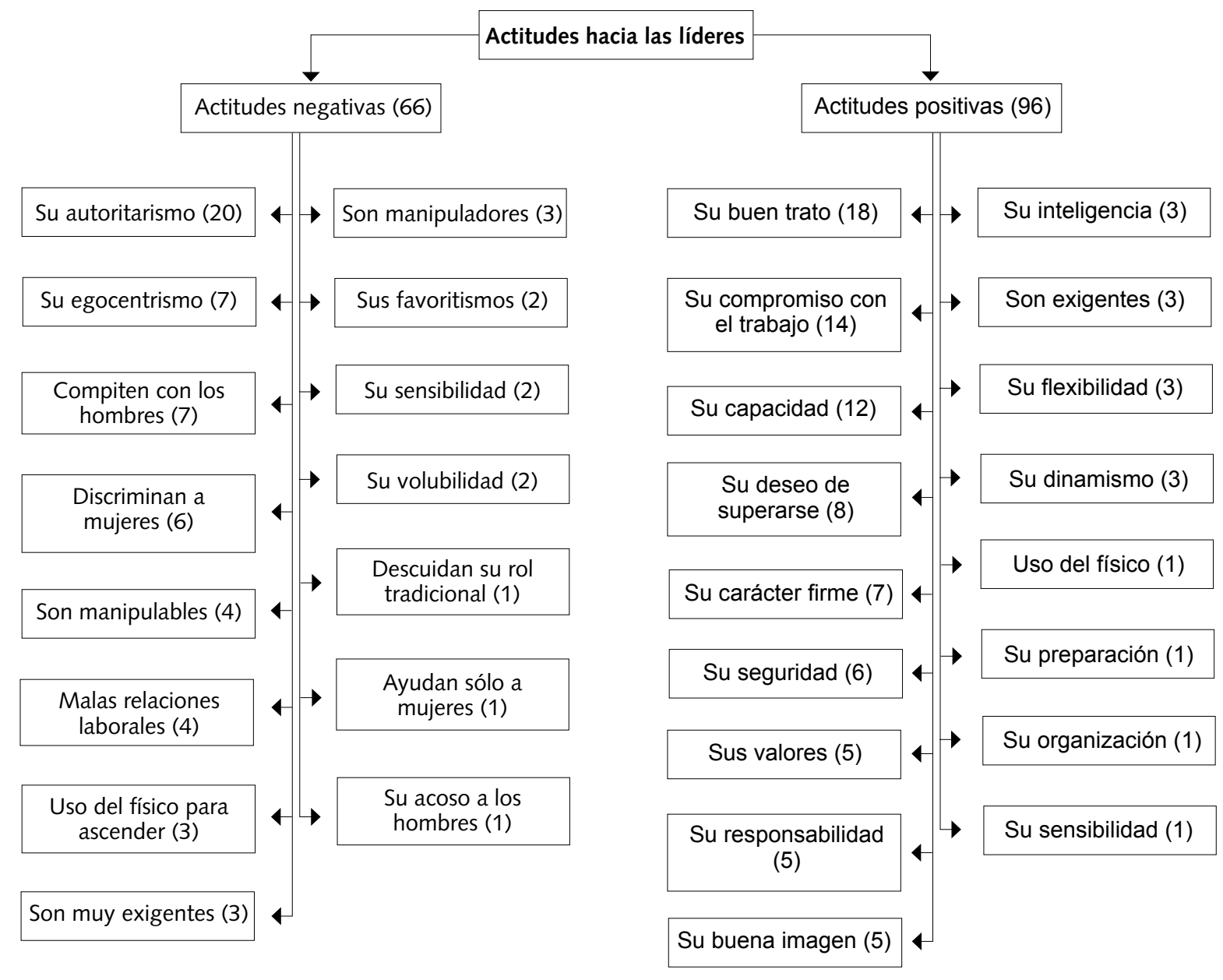

\section{DISCUSIÓN}

Al explorar las creencias y actitudes hacia líderes de ambos sexos, con el fin de contextualizar la medición del sexismo en escenarios laborales de México, se encontró que existen tanto creencias positivas como negativas hacia las líderes, vinculadas con sus roles tradicionales de género y, de manera importante, con los roles tradicionales masculinos. Al respecto parece corroborarse lo planteado por Díaz-Guerrero (1990), al señalar que las diferencias entre hombres y mujeres en relación con la masculinidad y la femineidad tienen un claro sustrato sociocultural, siendo sensibles a lo que en cada cultura en determinado momento histórico es delimitado como "masculino" o "femenino", y que en la actualidad, dados los cambios en las actividades desarrolladas por hombres y mujeres, permiten la flexibilización de los roles de género. Sin embargo, resulta de interés que las creencias negativas y positivas que recibieron un mayor número de menciones sobre las líderes, son aquellas identificadas con aspectos relacionales o afectivos, y esto sugiere que, en el contexto de nuestra cultura, dicha evaluación sigue girando en torno a un conjunto de roles y rasgos asociados a la feminidad tradicional, lo que puede evidenciar la prevalencia de la rigidez cultural aun en la actualidad. Por otra parte, es posible que el hecho de que las mujeres reci- 
ban más menciones en términos de creencias positivas que negativas muestre las implicaciones favorables que han tenido las políticas de equidad de género, al menos en el nivel de las creencias como lo señalan Eagly y Mladinic (1994); en tanto dichas políticas han propiciado evaluaciones más favorables hacia las acciones vinculadas a un liderazgo relacional. Sin embargo, convendría señalar que algunas de estas menciones se vinculan específicamente con el sexismo benevolente hacia las mujeres, planteado por Glick y Fiske (1996), y con la percepción de congruencia con el rol de género tradicional femenino (Eagly \& Karau, 2002), ya que, como se aprecia en los resultados las menciones positivas más frecuentes para las líderes, fueron las que hablan de las buenas relaciones en el trabajo.

En el caso de los hombres líderes, se percibe que los trabajadores los identifican más con categorías de tipo negativo que positivo. Aunado a ello, las características negativas se asocian específicamente a lo congruente con el rol de género tradicional masculino en el plano de lo autoritario y del abuso del poder, lo que, de acuerdo a Glick y Fiske (1999), pone de manifiesto el sexismo hostil hacia los hombres. La identificación con lo tradicionalmente masculino se conserva en la descripción de las características positivas, ya que no se mencionaron aspectos vinculados a categorías del tipo femenino tradicional. Al parecer, la creencia de los trabajadores es que los hombres se han mostrado resistentes al cambio en el plano de lo positivo y lo negativo, como señala Montesinos (2005). Por otra parte, el único aspecto que resalta de las creencias negativas sobre líderes, y que no sería congruente con lo indicado por Glick y Fiske (1999), es el vinculado al cuidado excesivo del físico, es decir, la metrosexualidad. Esto podría explicarse en función de la devaluación de lo "femenino" en los contextos de ejercicio del poder, ya que quizá para los trabajadores un hombre que cuida su apariencia en exceso parece estar más preocupado por la imagen que por el trabajo en sí mismo.

De las actitudes hacia los líderes, se presentó un equilibrio entre la evaluación favorable y desfavorable de los mismos. En cuanto a las evaluaciones desfavorables, los resultados de este estudio parecen dar sustento a los planteamientos de Glick y Fiske (1999), en el sentido de que se presenta un sexismo hostil hacia los hombres sólo en el plano de la masculinidad negativa.
En el mismo sentido, los elementos que generan una actitud positiva hacia los líderes también se asocian a la masculinidad positiva, como lo plantea el sexismo benevolente; es decir, la pauta de la predisposición actitudinal sexista hacia los hombres resulta de la identificación lineal entre el "ser hombre" y las conductas o características de dominio esperadas en él, o congruentes con su rol de género tradicional, teniendo en cuenta que las premisas culturales que delimitan la identidad masculina son la fuente elicitadora de dicho prejuicio.

Resulta de interés que las categorías con mayor número de menciones dentro de las actitudes negativas hacia las líderes sean aquellas integradas en el rol de género masculino negativo; es decir, a los trabajadores les disgusta más que una mujer desempeñe las actividades negativas asociadas estereotípicamente a los hombres, que incluso las actividades negativas asociadas estereotípicamente a las mujeres. En este sentido, autoras como Eagly y Karau (2002) señalan que el liderazgo en sí mismo es una actividad vinculada al estereotipo masculino, por lo que, de alguna forma, los trabajadores recurren a dicho estereotipo como su único elemento evaluativo. Además, estas autoras señalan que la percepción de incongruencia de roles lleva a exacerbar los afectos negativos hacia la persona evaluada, independientemente de su sexo; no obstante, conviene acotar el planteamiento y señalar que en el contexto laboral mexicano sólo las líderes incongruentes con su rol de género tradicional son evaluadas de una forma más negativa.

Por otra parte, aún se sigue teniendo disgusto por las mujeres líderes en función de creencias estereotípicas sexistas, tales como: "las mujeres no saben trabajar en equipo", "las mujeres son manipulables" o "usan su físico para ascender", ideas que se han manifestado en estudios como el de García-Retamero y López-Zafra (2006) y que se corroboran en la presente investigación. Por último, comienza a vislumbrarse una problemática propia a las interacciones actuales entre hombres y mujeres en el mercado de trabajo, haciéndose evidente un proceso de competitividad asociada al rechazo del crecimiento del otro, lo cual se refleja en las siguientes frases: "a los hombres les disgusta que las mujeres quieran ganarles", "a los trabajadores les disgusta que las líderes descuiden su rol tradicional" y "a 
los trabajadores les disgusta que las líderes acosen a los hombres".

En términos de los aspectos que generan actitudes positivas hacia las líderes, se encontró que su flexibilización en los roles, es decir, tener características positivas tanto masculinas (firmeza de carácter) como femeninas (buen trato) lleva a una evaluación favorable de dichas líderes. Al respecto, autores como Rocha y Díaz-Loving (2005) señalan que la incorporación de la mujer al campo laboral en las últimas tres décadas está permeando lentamente la visión estereotipada sobre los géneros, lo cual es congruente con el impacto que estas transformaciones tienen en el desempeño de roles menos tradicionales y que apuntan a aspectos vinculados a una androginía positiva, entendida como la capacidad de un individuo para mostrar tanto rasgos como comportamientos instrumentales y expresivos. Esto, además, resulta coherente con los planteamientos actuales del liderazgo en los que se señala que debe existir una adecuada interacción entre las actividades orientadas a la tarea y a las relaciones (Terroni, 2002).

En conclusión, los resultados de este trabajo parecen ser consistentes con la literatura relacionada al estudio del sexismo en la actualidad. Se necesita indagar en las creencias y actitudes que dan paso a la discriminación sexista, ya que, como lo señalan Glick y Fiske (1996, 1999), la ambivalencia que generan los estereotipos y actitudes hacia los roles de género en términos de lo positivo y lo negativo, tiene repercusiones en muchos ámbitos del desarrollo humano, dentro de los cuales se pudo corroborar que el liderazgo empresarial no es la excepción. La principal contribución de la presente investigación radica en significar cómo la percepción de congruencia del rol de liderazgo con el rol de género tradicional (Eagly y Karau, 2002) delimitan y propician evaluaciones que, bajo un contexto cultural rígido, podrían llevar a un sexismo sutil o benevolente en las organizaciones, el cual puede afectar de la misma forma que el sexismo hostil. Se sugiere que esta ambivalencia del sexismo tiene repercusiones negativas en el logro de la equidad de los géneros dentro de los puestos de liderazgo en las empresas, como lo muestran los datos del INEGI (2007) y del FEM (2009). También es importante resaltar que no todas las creencias y predisposiciones actitudinales hacia los líderes fueron sexistas, lo cual podría representar un área de oportunidad para enfatizar que el liderazgo en las organizaciones se debe orientar a la integración de cualidades que pueden ser aprendidas por ambos sexos y que no son exclusivas de uno u otro.

Futuras investigaciones deben tomar en cuenta la inclusión de organizaciones del sector servicios e incrementar el tamaño de la muestra con empleados de distintas regiones de nuestro país, ya que esto se constituyó como un aspecto limitante de la presente investigación, al no poder generalizar los resultados al ámbito laboral mexicano. Asimismo, se sugiere que a partir de los resultados de esta investigación pueden desarrollarse inventarios de sexismo que retomen la perspectiva del sexismo ambivalente de Glick y Fiske $(1996,1999)$, en concordancia con la teoría de la congruencia del rol de género de Eagly y Karau (2002), de modo que dichos inventarios sean sensibles a las características culturales y específicas al área laboral.

\section{REFERENCIAS}

Biglia, B. (2003b). Transformando dinámicas generizadas: Propuestas de activistas de movimientos sociales mixtos Athenea Digital 4, 1-25.

CNN Expansión. (2010). Mujeres dirigen sólo el 3\% de las 500. Recuperado el 10 de agosto de 2010 de http://www.cnnexpansion.com/las-500-de-expansion/2010/06/10/las-mujeres-lideran-solo-3-delas -500
Díaz-Guerrero, R. (1990). ¿Existen rasgos básicos en la personalidad de los mexicanos? Revista Mexicana de Psicología, 7 (1-2), 121-129.

Eagly A. H. \& Johnson, B. T. (1990). Gender and leadership style: A meta-analysis. Psychological Bulletin, $108,233-256$

Eagly, A. H. \& Karau, S. J. (2002). Role congruity theory of prejudice toward female leaders. Psychological Review, 109, 573-598. 
Eagly, A. H., \& Mladinic, A. (1994). Are people prejudiced against women? Some answers from research on attitudes, gender stereotypes and judgments of competence. In W. Stroebe \& M. Hewstone (Eds.). European Review of Social Psychology (Vol. 5, pp. 1-35). New York: Wiley.

Fernández Poncela, A.M. (2000). Mujeres, revolución y cambio cultural. Barcelona: Anthropos.

Foro Económico Mundial (2009). Indice de Disparidad de Géneros. Recuperado el 10 de septiembre de 2010 de http://www.weforum.org/pdf/gendergap/ report2009.pdf

García-Retamero, R., y López-Zafra, E. (2006). Congruencia de rol de género y liderazgo: el papel de las atribuciones causales sobre el éxito y el fracaso. Revista Latinoamericana de Psicología, 38, 245-257.

Glick, P., Diebold, J., Bailey-Werner, B., \& Zhu, L. (1997). The two faces of Adam: Ambivalent sexism and polarized attitudes toward women. Personality and Social Psychology Bulletin, 23, 1323-1334.

Glick, P., \& Fiske, S. T. (1996). The Ambivalent Sexism Inventory: Differentiating hostile and benevolent sexism. Journal of Personality and Social Psychology, 70 (5), 491-512.

Glick, P., \& Fiske, S. T. (1999). The Ambivalence toward Men Inventory: Differentiating hostile and benevolent beliefs about men. Psychology of Women Quarterly, 23(3), 519-536.

Grooser, K. \& Moon, J. (2005). Gender Mainstreaming and Corporate Social Responsibility: Reporting Workplace Issues. Internacional Centre for Corporate Social Resposibility. 27, pp.1-34

Instituto Nacional de Estadística, Geografía e Informática. (2007). Resultados de la Encuesta Nacional de Ocupación y Empleo. Recuperado el 9 de febrero de 2009 de http://www.inegi.org.mx/inegi/contenidos/ espanol/prensa/Boletines/Boletin/Comunicados/ Especiales/2007/Junio/comunica6.doc

Izquierdo, María Jesús (2003), Contenidos y significados de nuevas formas de cuidado, SARE 2003, "Cuidar cuesta: costes y beneficios del cuidado", Emakunde.

Montesinos, R. (2005). Feminidades y masculinidades emergentes. Del espacio público al privado y viceversa, Veredas (10), Primer semestre.
Rocha, S. T. y Díaz-Loving, R. (2005). Cultura de género: La brecha ideológica entre hombres y mujeres. Anales de Psicología, 21(1), 42-49.

Ryan, M. K., Haslam, S. A., Hersby, M., \& Bongiorno, R. (2007). Think crisis-think female: Glass cliffs and contextual variation in the think manager-think male stereotype. Working paper, University of Exeter

Swim, J. K., Aikin, K. J., Hall, W. S., \& Hunter, B. A. (1995). Sexism and racism: Old fashioned and modern prejudices. Journal of Personality and Social Psychology, 68, 199-214.

Terroni, N. (2002). El liderazgo en grupos y organizaciones: Una revisión teórica. Revista IDEA, 37 75-84.

Zabludovsky, G. (2007). México: Mujeres en cargos de dirección del sector privado. Academia, Revista Latinoamericana de Administración, 38, 9-26.
Fecha de recepción: Abril 2011 Fecha de publicación: Diciembre 2011 